\title{
Side-impact collisions of Ar with NO
}

Cornelia G. Heid ${ }^{1 \sharp}$, Victoria Walpole ${ }^{1 \sharp}$, Mark Brouard ${ }^{1 *}$, Pablo G. Jambrina ${ }^{3 \ddagger}$, and F. Javier Aoiz ${ }^{2 \natural}$

${ }^{1}$ The Department of Chemistry, University of Oxford, The Chemistry Research Laboratory, 12 Mansfield Road, Oxford, OX1 3TA, United Kingdom.

${ }^{2}$ Departamento de Química Física, Facultad de Química, Universidad Complutense, 28040 Madrid, Spain.

${ }^{3}$ Departamento de Química Física, Universidad de Salamanca, 37008, Salamanca, Spain

*To whom correspondence should be addressed; E-mail: mark.brouard@chem.ox.ac.uk

E-mail addresses: ${ }^{\ddagger}$ pjambrina@usal.es, ${ }^{\natural}$ aoiz@quim.ucm.es

$\sharp$ These two authors contributed equally. 
In the realm of molecular collision dynamics, stereochemistry refers to the dependence of the collision outcome on the mutual orientation of the colliding partners. This may involve directed end-on collisions along a molecular bond-axis or encounters in which the partner approaches the bond of an oriented molecule from the side. Using both experiment and theory, we show here that in the simplest case of an inelastic collision between an atom and a nearly homonuclear diatom, in which the two atoms have almost the same mass, the scattering dynamics are very distinct for impacts on either side of the molecule. By recording the direction of the scattered particles after collision, we demonstrate that the intensity of products scattered in the forward direction, near parallel to the initial motion, can be substantially controlled and even maximised, by altering the side-on orientation of quantum state selected NO molecules colliding with Ar atoms. In addition, our findings provide valuable information about the preferred collision mechanism and reveal interesting quantum interference effects.

The concepts of the steric effect and the steric factor have their foundations in chemistry going back to early studies of kinetic theory over a hundred years $\mathrm{ago}^{1}$, and the idea that the outcome of molecular collisions might be controlled by orienting collision partners in particular directions continues to capture the imagination of chemists to this day. This article considers collisions between an Ar atom and the nitric oxide molecule (NO), in which the collision energy is transferred into rotational excitation of the scattered NO. Here we show that by first quantum state selecting NO in its lowest rotational state, and then orienting its bond-axis, $r$, preferentially side-on to the incoming Ar atom, using a static electric field, we can provide exquisite control of the direction of scattering after collision. In fact, we show that the intensity of products scattered in the forward direction, near parallel to the initial motion, can be substantially controlled and even maximised by altering the side-on orientation of the NO molecule. We also demonstrate that the ability to measure the variation of the collision cross-section with initial molecular orientation 
provides valuable insight into the preferred scattering mechanism.

There has been considerable interest in elucidating how the initial molecular axis direction, $r$, influences the outcome of inelastic and reactive collisions, and, complementary to that, the role of the direction of the initial molecular rotational angular momentum, $j^{2-8}$. Studies have primarily focussed on investigating the effects of 'head-on' versus 'side-on' alignment of the incoming molecules on the collisional process (see Supplementary Fig. 1) ${ }^{4,7,9-11}$. The effect on the collision dynamics of end-on 'heads versus tails' molecular orientation, parallel or antiparallel to the approach direction, has also been the subject of several studies ${ }^{3,4,7,12-14}$. By contrast, very little attention has been paid to the effect of initial side-on bond-axis orientation on the scattering process, in which the direction of approach is preferentially perpendicular to the oriented molecular axis ${ }^{15,16}$. Although in crossed molecular beam experiments the impact parameter (i.e. the distance of closest approach in the absence of an interaction potential) cannot be selected, intuitively, one might expect that side-on oriented collisions will be more sensitive to higher impact parameter collisions, leading to more forward scattering (i.e. NO scattering in the same direction as the initial NO motion, $\theta<90^{\circ}$ ), than those occurring in an end-on configuration. The latter are expected to be more dominated by lower impact parameter (head-on) collisions, preferentially leading to backward scattered trajectories $\left(\theta>90^{\circ}\right)$. Hence, to obtain a complete picture of the collision dynamics, it is necessary to probe both end-on and side-on collisions: the work described here provides a route to exploring such effects directly experimentally and theoretically.

The inelastic scattering of $\mathrm{NO}$ with the rare gases has been used to demonstrate complete quantum state resolution in studies of inelastic scattering ${ }^{17-21}$, with experiments now affording sufficiently high resolution to reveal fascinating quantum diffraction and resonance structures in the differential cross sections (DCSs) - or angular distributions - of the scattered products ${ }^{16,22,23}$. In addition, $\mathrm{NO}+\mathrm{Ar}$ was the first system in which the effects of end-on bond-axis orientation were 
studied with full quantum state resolution ${ }^{12,14,24,25}$. For these end-on collisions one can control the intensity of scattering into the backwards direction, but forward scattering is relatively less affected. In contrast to previous work in which end-on orientation was considered ${ }^{12,14,24,25}$, here we preferentially orient $\mathrm{NO}$ side-on to the incoming Ar atom, perpendicular to the initial relative velocity vector, $\boldsymbol{k}$, and monitor the direction of scattering using resonantly enhanced multiphoton ionisation (REMPI) coupled with velocity map ${ }^{26}$ ion-imaging ${ }^{27}$ (VMI).

Figure 1 illustrates key features of the experiment. Panel a defines the two scattering frame side-on orientations, labelled $\pm x$, considered in this work, whilst panels $\mathrm{b}$ and $\mathrm{c}$ illustrate the experimental configuration employed. Because of the open shell nature of the $\mathrm{NO}\left({ }^{2} \Pi_{\Omega}\right)$ ground electronic state, each rotational state is split into two $\Lambda$-doublet levels, labelled $e$ and $f$. We use a hexapole electric field to initially select just the upper low-field-seeking $f$ component of the lowest $\left(\Omega=1 / 2, j=1 / 2\right.$ ) rotational quantum state of $\mathrm{NO}{ }^{12}$. Here, $j$ and $j^{\prime}$ refer to the initial and final quantum numbers for the total angular momentum of NO apart from nuclear spin. $\Omega$ is the absolute value of the quantum number for the projection of the electronic angular momentum along the internuclear axis, which defines the spin-orbit state of the $\operatorname{NO}\left(X^{2} \Pi_{\Omega}\right)$ molecule. On exiting the hexapole, the state selected molecules are adiabatically oriented along a chosen axis by applying a static electric field, $\boldsymbol{E}^{3,24,25}$, created by a set of four rods, as shown in Fig. 1b. In the static field, the initial $\Lambda$-doublets of the NO molecule become mixed, so that scattering with $\mathrm{Ar}$ takes place with $\mathrm{NO}$ in a coherent superposition of the two lowest $e$ and $f$ levels ${ }^{7,12}$. Under these conditions, the NO bond-axis, $\boldsymbol{r}$, becomes oriented about the field direction, $\hat{\boldsymbol{E}}$, according to the distribution shown in the inset of Fig. 1a. The $\mathrm{N}$ atom of the NO molecule preferentially points towards the negative electrode, such that the bond-axis, $r$ (which coincides with the direction of the permanent dipole moment, $\mathrm{N} \rightarrow \mathrm{O}$ ), preferentially points in the direction opposite to the field, as illustrated in Fig. 1a-c. In the figure, we show the bond-axis in its most probable orientation, antiparallel to the field direction, but in reality $\boldsymbol{r}$ is distributed about $\boldsymbol{E}$, as shown in the inset to 
Fig. 1a.

The experiments reported here focus on the $\Omega^{\prime}=1 / 2$ spin-orbit state of the scattered NO in the $e$ final $\Lambda$-doublet level. Because of the mixing of the initial $\Lambda$-doublet levels in the static electric field, similar results are found for the $f$ final $\Lambda$-doublet level. Note that, at the static electric fields employed, the $\Lambda$-doublet levels of the excited NO rotational states populated after collision are not significantly mixed.

\section{Results}

Representative ion-image data, obtained probing the final e $\Lambda$-doublet levels of $\operatorname{NO}\left(\Omega^{\prime}=1 / 2\right)$ for $\Delta j=j^{\prime}-1 / 2=7$ to 12 are presented in Fig. 2 for orientation of NO in the $+x$ (panels a) and $-x$ configurations (panels $\mathrm{b}$ ). These particular final rotational states represent a subset of the data we have collected and are chosen here because they most prominently exhibit the steric effects discussed in the following. Because the static field direction for each of the raw images shown is fixed in the experiments, scattering intensity in different portions of the image (e.g., the bottom left versus the top right of the images) actually correspond to different scattering frame orientations of the field. However, due to experimental factors affecting the detection sensitivity across the image, such as the flux-density transformation, the most intense region of the images corresponds to in-plane scattering into the lower left region of the image. This portion of the image is indicated in Fig. 1c as the brighter, unshaded region, and corresponds to slow-moving scattered NO in the laboratory. For this reason we label the experimental ion image configuration, $\pm x$, according to the field orientation with respect to scattering into this more intense half of the image (see Supplementary section 1.3.1).

Whilst the NO orientation is controlled through application of the static electric field, and the Ar atom preferentially approaches NO from the side, only for the smallest impact parameters 
does Ar actually hit the side of NO close to its centre-of-mass. In most collisions, Ar hits the side of the NO molecule at (or towards) either the $\mathrm{N}$ atom or the $\mathrm{O}$ atom in higher impact parameter collisions, as illustrated in the bottom panels of Fig. 2 and Supplementary Fig. 1. We refer to these collisions as $\mathrm{N}$-side or $\mathrm{O}$-side, respectively. Although these side-on collisions generally involve higher impact parameters than in the end-on configuration, for the transitions studied here scattering is dominated by the repulsive core of the potential energy surface (PES), rather than the long-range attractive region, which really only dominates for low rotational excitations $(\Delta j \leq$ $3)^{28}$. Given that the orientation direction $( \pm x)$ is defined in terms of the field direction, $\hat{\boldsymbol{E}}$, the $+x(-x)$ orientation therefore corresponds to repulsive collisions preferentially off the $\mathrm{N}$-side $(\mathrm{O}-$ side) of NO, as illustrated in Fig. 1a. We note that these cartoons provide a simple and intuitive (classical) picture. Quantum mechanically, the outgoing direction of the Ar atom may arise from interfering trajectories on either side of the molecule.

The relative intensities of the images shown in the $\pm x$ orientations in Fig. 2 reflect the signals recorded in the two geometries, and exhibit a strong alternation between $+x$ and $-x$ for $\Delta j$ odd and even, respectively. This intensity alternation represents an integral $x$-axis steric effect, which is particularly pronounced for the $\Delta j$ shown here. Furthermore, the features in the raw ion images are shown also to be strongly dependent on the $+x$ or $-x$ orientation. Strikingly, we find that the raw images for transitions involving odd changes in rotational quantum state display intense scattering in the forward direction (i.e. small angle scattering of NO with respect to its initial motion) and a pronounced double peaked structure when the Ar approaches the side of the NO molecule and repulsively scatters off the $\mathrm{N}$-side ( $+x$ orientation), whereas only a single peak is observed when $\mathrm{Ar}$ approaches the side of NO and repulsively scatters off the O-side of the molecule ( $-x$ orientation). By contrast, even $\Delta j$ transitions are largely dominated by just a single strong maximum in their images for both bond-axis orientations. 
Figure 2 also presents difference ion images, obtained by subtracting the images in panels $b$ from those in panels a. The difference images highlight the field- or orientation-dependence of the scattering process. The experimental difference images show a sign inversion around the initial relative velocity vector, reflecting the fact that scattering into the bottom left and top right of the image corresponds to different scattering frame orientations of the field. Note that if it were not for experimental factors associated with differing detection sensitivity across the image, these difference images would be perfectly anti-symmetric about the relative velocity vector (see Supplementary section 1.3.1). The experimental difference image data (panels 2c) are compared with the results of a full simulation of the difference images obtained using new quantum mechanical (QM) scattering calculations (panels d), on the potential energy surfaces (PESs) by Alexander ${ }^{29}$. The calculations take full account of the side-on orientation of NO in the static electric field, and the simulations also allow for the experimental detection sensitivity factors mentioned above. The agreement between the experimental and simulated difference images is generally excellent.

The ion images can also be fitted to obtain the angular distribution of the scattered products for the different initial bond-axis orientations ${ }^{21,24,30}$. The resulting experimental angular distributions (blue continuous lines in panels e and f of Fig. 2) are directly compared with the results of the QM scattering calculations (red dashed lines). The experimental angular distributions are scaled to preserve the observed relative intensities in the $\pm x$ orientations. As with the difference images, the agreement between experiment and QM theory is also very good, with the relatively small differences between experiment and theory generally within the experimental uncertainties, and serves to validate the theoretical procedures developed here and further confirms the accuracy of PESs employed ${ }^{29}$.

The DCSs shown in Fig. 2e-f display the striking trends already apparent in the raw images: the DCSs for odd $\Delta j$ transitions for the $+x$ bond-axis orientation display pronounced scattering 
in the forward direction, favouring repulsive scattering off the $\mathrm{N}$-side of the molecule. For example, the odd $\Delta j$ transitions shown approach a nearly ten-fold enhancement in scattering intensity around the peak in the forward direction for the $+x$ orientation compared with that in the $-x$ configuration. Furthermore, the DCSs for these odd $\Delta j,+x$-oriented transitions exhibit multiple peaks, corresponding to the structures observed in the raw ion images. The intense forward peak in the DCSs (highlighted by the white dashed circles on the relevant images in panels a) is almost completely suppressed when the field is oriented such that Ar repulsively scatters off the O-side of the molecule ( $-x$-direction). However, for even $\Delta j$ transitions the picture is reversed, with the intense forward peak observed for scattering off the O-side of the molecule ( $-x$-orientation), but multiple peaks are absent, and the relative intensities of the forward peaks in the $\pm x$ geometries is less pronounced than for the odd $\Delta j$ transitions. For the highest rotational excitations ( $\Delta j \geq 12$ ), the two orientations lead to similar DCSs, with a modest preference for scattering in the $+x$ orientation. This behaviour can be rationalized by the relatively low impact parameters required for these higher rotational excitations; as the PES on the side of the molecule, close to the centre-ofmass (i.e. at low impact parameters), varies only slightly, collisions to either side result in similar scattering dynamics.

Three factors are primarily responsible for the detailed form of the DCSs shown in Fig. 2 (see also Supplementary section 2). Firstly, the strength of the static electric field determines the mix of the initial $e$ and $f \Lambda$-doublet levels in the initial state and the degree of bond-axis orientation ${ }^{24,25}$. This directly affects the relative intensities of the peaks in the DCSs. At low fields the DCSs resemble those for the field free parity-resolved $f \rightarrow e$ transitions, irrespective of field orientation. Due to parity effects, these have been shown previously to possess multiply peaked DCSs for odd $\Delta j$, but only single peaks for even $\Delta j^{21,28}$. At the limit of infinite field, when the initial $\Lambda$-doublet states are fully mixed with equal weights in the superposition state, multiple peaks are observed for all $\Delta j$ transitions in the DCSs for the $+x$-orientation, but only single peaks in the $-x$ - 
orientation (for reasons discussed below). At the experimental field one finds a somewhat more intermediate picture, because the initial $\Lambda$-doublet states are only partially mixed, with the initial $f$ state contributing about two thirds to the superposition. For this reason, at the experimental field employed, multiple peaks are seen most clearly only for odd $\Delta j$ in the $+x$-orientation, rather than for all $\Delta j$.

The second factor to consider is the role of rotational rainbows in determining the form of the $\pm x$ resolved DCSs at infinite field. We have applied a simple semi-classical hard-shell scattering model ${ }^{21,28,30}$ which accounts qualitatively for the infinite field scattering behaviour discussed above. The model demonstrates that the multiple peaks seen in the infinite field DCSs for the $+x$-orientation can be associated with QM rotational rainbow structures arising from interfering trajectories repulsively scattering off the side and the N-end of NO. For the $-x$ orientation, associated with interfering trajectories repulsively scattering off the side and the O-end of $\mathrm{NO}$, the phase shift between the side- and end-on trajectories is smaller than for the $+x$ orientation, because the repulsive core of the PES protrudes less on the O-end of the molecule than on the $\mathrm{N}$-end. The greater phase shift generated for the $+x$ orientation than for the $-x$ orientation leads to more peaks in the associated DCSs for the former configuration. To further validate this argument, we have also performed QM scattering calculations in which we have swapped the masses of the $\mathrm{N}$ and $\mathrm{O}$ atoms, obtaining more peaks for collisions towards the side of the lighter atom, just as would be expected from the simple semi-classical treatment.

Finally, QM interference between the coherently prepared $\Lambda$-doublet levels plays a vital role in determining the relative intensities of the scattering distributions in the $\pm x$ orientations ${ }^{14}$. This QM interference can be traced back to the relative phase shift of the scattering amplitudes associated with transitions from the initial $e$ and $f \Lambda$-doublet states to a given final state. The sign of this phase shift changes between odd and even $\Delta j$ transitions, which, in turn, leads to the character- 
istic alternation of the total scattering intensity between adjacent $\Delta j$ transitions. Specifically, this QM interference leads to an enhancement in intensity for the $+x$ orientation when $\Delta j$ is odd, and an enhancement in intensity for the $-x$-orientation when $\Delta j$ is even, as clearly seen in the data presented in Fig. 2. Because QM interference determines the relative intensities in $+x$ and $-x$ orientations (i.e. the integral steric asymmetry effect), it also ensures that the double peaks in the DCSs are observed particularly strongly for odd $\Delta j$, and also determines the distinctive forms of the difference ion images for even versus odd $\Delta j$.

\section{Discussion}

We highlight here two aspects of the present study which are of particular significance, namely the dramatic control that $x$-axis orientation is able to exert on the intensity of forward scattering, and the new insight the measurements provide about the scattering mechanism.

As Ar approaches NO from the sides, it sees a different potential energy landscape for $\mathrm{N}$ - and O-side encounters, and the direction of scattering is thus highly sensitive to the side-on bond-axis orientation. It is precisely this ability to differentiate between different approach configurations that allows us to control the scattering in the forward direction in this geometry. Here we have demonstrated that, at the experimental field strengths employed, $x$-axis orientation can lead to an enhancement in scattering intensity of a factor of almost ten (see Fig. 2). Such strong control cannot be achieved if the NO molecule is oriented in the end-on configuration, for which the topography of the potential is more symmetric around the molecular axis.

The degree of control achieved for side-on and end-on orientations, along the $x$ - and $z$-axes, respectively, is illustrated in Fig. 3. The left (a) and right (b) panels of the figure compare the calculated DCSs obtained in the $x$ - and $z$-axis orientations at infinite field with the maximised DCSs for $\Delta j=7$ and 12 (shown as the white area). The maximised DCS corresponds to the 
maximum DCS obtained at each scattering angle for any arbitrary orientation of the static electric field, $\boldsymbol{E}$, and hence any arbitrary orientation of the bond-axis. From this comparison it is possible to ascertain the control that can be achieved at a given scattering angle by optimal choice of static field direction. In the forward hemisphere, the maximum DCS is very close to that achieved with $\pm x$ orientation (Fig. 3a), particularly so for $\Delta j=7$, whereas for scattering in the backward hemisphere $\left(\theta>90^{\circ}\right)$ the degree of control in the $\pm x$ orientation is less pronounced. By contrast, NO orientation along the $z$-axis (Fig. 3b) leads to modest selectivity in the forward region, but is close to the maximum for backward scattering, as is particularly striking for $\Delta j=12$.

A key feature of our experiment is that it helps elucidate the scattering mechanism. Fig. 4a compares the calculated infinite field DCSs assuming an isotropic bond-axis distribution for the coherent state produced within the static electric field, equivalent to the average of the $+x$ and $-x$, or $+z$ and $-z$, oriented DCSs (shown as the orange line) with the maximised DCSs for $\Delta j=7$ and 8 (shown as the white area). The $+x$ and $-x$ oriented DCSs are also included. The data further emphasize the importance of $x$-axis orientation in controlling scattering into the forward region. At a given scattering angle, the preferred mechanism which maximises the DCS is shown in Fig. 4 b for $\Delta j=7$ and 8 , with the shaded angular distributions reflecting how the DCSs at a given scattering angle are influenced by the initial bond-axis orientation. Our experimental results show that forward scattering is enhanced with $x-$ (side-on) approach whilst backward scattering is nearly maximized with end-on $(z-)$ approach. Strikingly, for the two transitions shown, the preference for $\mathrm{O}$-side or $\mathrm{N}$-side collisions is reversed. As already mentioned, this behaviour arises from QM interference between the scattering amplitudes of the initially prepared coherent mixture of $\Lambda$-doublet states, and cannot be explained within a classical framework ${ }^{14,31}$.

Our results demonstrate that the ability to orient molecules in different directions can yield new insight about the scattering mechanism, as illustrated here in Fig. 4. We have shown that this 
information can be determined directly both from the experiment and from the newly extended theory. By varying the bond-axis orientation, one can to some extent also control the contributions from different impact parameters, and ultimately the collision outcome. In addition, the possibility of modeling the collision dynamics in arbitrarily oriented electric fields presents a powerful tool to elucidate the steric preferences of scattering systems, and should stimulate the design of novel experiments. For example, in the context of molecular scattering it would be particularly interesting to look at orientation effects in more complex systems, such as molecule-molecule scattering. Extensions to the observation of bond-axis orientation effects in reactive systems would also be of great interest, and potentially complement measurements made using initial state alignment induced by polarised light.

\section{Methods}

Experimental methods The experiments were carried out in a crossed-molecular beam apparatus, with the NO (10\% in Ar) and Ar beams, at backing pressures of 3 and 2 bar respectively, crossing at right angles in the centre of the scattering chamber, as described previously ${ }^{24,25}$. The NO beam was expanded using a General valve (Series 9$)$ and skimmed $(d=3 \mathrm{~mm})$ before entering a hexapole state selector, where the low-field seeking $f \Lambda$-doublet level of the $\mathrm{NO}(\mathrm{X}, v=0, j=1 / 2, \Omega=$ $1 / 2$ ) rotational state was selected and focussed. The Ar beam was expanded through a Jordan valve and also skimmed $(d=3 \mathrm{~mm})$. Prior to collision with Ar, the NO molecules were oriented in a static electric field of $9.2 \mathrm{kV} / \mathrm{cm}$. This was created by a set of four rods placed in the interaction region, with the two rods on opposite sides charged to $+8 \mathrm{kV}$ and $-8 \mathrm{kV}$ (see Fig. 1b). The electric field was oriented perpendicular to the relative velocity vector of the two beams $\left(\boldsymbol{k}=\boldsymbol{v}_{\mathrm{Ar}}-\boldsymbol{v}_{\mathrm{NO}}\right)$, such that the Ar approached the NO preferentially from the side (Fig. 1b and c). The direction of the electric field was switched every 200 laser shots to change the orientation of the NO molecules, thus minimising the effect of experimental drift on the measurement. The NO primary beam was 
operated at $10 \mathrm{~Hz}$, whilst the secondary Ar beam was operated at $5 \mathrm{~Hz}$ to allow for background subtraction on a shot-to-shot basis. For a given orientation, including background shots, the ion images were typically converged after about $\lesssim 10^{5}$ laser shots. In all measurements, the mean collision energy was determined to be $651 \mathrm{~cm}^{-124,25,32}$.

Following a fixed delay after the molecular beam pulses, the scattered NO molecules were state selectively ionised using a $\left(1+1^{\prime}\right)$ REMPI scheme via the NO(A) state ${ }^{24,25}$. The first photon was supplied by a frequency-doubled dye laser in the region around $226 \mathrm{~nm}$, and the second photon by a $\mathrm{XeCl}$ excimer laser $(308 \mathrm{~nm})$. All data were recorded on the $Q_{21}+R_{11}$ (mixed) branch transition of NO, with the polarisation of the $226 \mathrm{~nm}$ light set to vertical with respect to the molecular beam plane using a linear polariser. The $308 \mathrm{~nm}$ ionisation laser radiation was unpolarised. The two laser beams were oriented at $90^{\circ}$ with respect to each other, and approximately $45^{\circ}$ with respect to the molecular beams, such that the probe beam propagating direction was close to perpendicular and the ionisation beam close to antiparallel to $\boldsymbol{k}$. Ions generated in the REMPI process were accelerated by a set of ion optics and velocity mapped onto a dual $\mathrm{MCP} /$ phosphor screen detector. The flashes on the phosphor screen were recorded with a charge-coupled device camera and transferred to a computer for data analysis. In order to achieve good velocity-mapping, Behlke switches were employed to rapidly switch the voltages on the rods from $\pm 8 \mathrm{kV}$ to about $1 \mathrm{kV}$. The time delay between the switching and the arrival of the probe laser beam in the interaction region was about $500 \mathrm{~ns}$, which was found to be short enough to retain orientation of the NO molecules.

Data analysis The image analysis is complicated by the fact that in the $x$-axis geometry scattering is no longer symmetric around the relative velocity vector. This means that the slow and fast side of the ion image in the laboratory frame (indicated in Supplementary Fig. 3), which reflect different directions of $\boldsymbol{k}^{\prime}$, correspond to opposite orientations with respect to the static field direction, $\boldsymbol{E}$, in the scattering frame. It is this break in symmetry of the scattered images which gives rise 
to the characteristic appearance of the difference images shown in Fig. 2. The experimental DCSs were extracted from the ion images by first determining the flux-density correction and then fitting the experimental sum and difference ion images to a linear combination of modified spherical harmonics basis functions by means of a genetic algorithm ${ }^{21,30}$. The flux-density correction was determined using a Monte Carlo procedure with the experimental parameters (such as detection volume, laser firing time, molecular beam velocity and temporal distributions, etc.) defining the space sampled during the simulation of the basis functions ${ }^{21,30}$. To account for the polarisation of the probe laser, a polarisation term, calculated from the kinematic apse model ${ }^{30,33}$, was included. The DCSs were calculated from the parameters obtained from the fitted images, whilst preserving the relative intensities of the data recorded in the $\pm x$ orientation. The simulations of the ion images employed the same basis set as in the fitting of the images, but used the initial bond-axis polarisation moments obtained from the QM calculations to express the intrinsic scattering distribution. A more in-depth discussion of the data analysis procedure can be found in Supplementary section 1.3 .

Theoretical methods The newly developed theory for modelling collisions of Ar atoms with NO molecules placed in an arbitrarily oriented static electric field is described in Supplementary section 1.2.1. The QM dynamical calculations were performed with the HIBRIDON suite of codes (http://www.chm.unipg.it/chimgen/mb/hibridon/index.html), which uses the log-derivative propagator of Manolopoulos. A propagation from $4.5 \mathrm{bohr}$ to $60 \mathrm{bohr}$ was used, and included a rotational basis with all $\mathrm{NO}(\mathrm{X})$ states up to $j=20.5$ and all partial waves up to total angular momentum quantum number, $J_{\max }=190.5$. These calculations used both the $V_{\text {sum }}=\left(V_{A^{\prime}}+V_{A^{\prime \prime}}\right) / 2$ and $V_{\text {diff }}=\left(V_{A^{\prime \prime}}-V_{A^{\prime}}\right) / 2$ potential energy surfaces (PESs) of Alexander ${ }^{29}$ (which assume a fixed bond length for the $\mathrm{NO}(\mathrm{X})$ molecule). Calculations were run over a grid of collision energies from $530 \mathrm{~cm}^{-1}$ to $740 \mathrm{~cm}^{-1}$ with a spacing of $35 \mathrm{~cm}^{-1}$, and the theoretical close-coupled QM DCSs were averaged over the appropriate experimental collision energy distribution. Although this has 
the effect of somewhat blurring the oscillations in the forward scattered direction at low values of $j^{\prime}$, the DCSs averaged over the distribution of collision energies is otherwise very similar to that obtained at the most probable collision energy.

Data Availability Experimental and computational data supporting the findings of this study are available from the Oxford Research Archive (ORA) (DOI: xxx).

Code Availability The Hibridon set of programs used for the QM calculations can be downloaded from http://www2.chem.umd.edu/groups/alexander/hibridon/hib43/index.html. The computer codes used for the data analysis are available from the corresponding author upon reasonable request. 


\section{References}

1. Trautz, M. Das Gesetz der Reaktionsgeschwindigkeit und der Gleichgewichte in Gasen. Bestätigung der Additivität von $C_{v}-3 / 2 R$. Neue Bestimmung der Integrationskonstanten und der Moleküldurchmesser. (The law of reaction rates and equilibria in gases. Confirmation of the additivity of $C_{v}-3 / 2 R$ : New determination of the integration constants and the molecular diameters.). Z. Anorg. Allg. Chem. 96, 1-28 (1916).

2. Levine, R. D. \& Bernstein, R. B. Molecular Reaction Dynamics and Chemical Reactivity (Oxford University Press, New York, USA, 1987).

3. Parker, D. H. \& Bernstein, R. B. Oriented molecule beams via the electrostatic hexapole: Preparation, characterization, and reactive scattering. Ann. Rev. Phys. Chem. 40, 561-595 (1989).

4. Loesch, H. J. Orientation and alignment in reactive beam collisions: Recent progress. Ann. Rev. Phys. Chem. 46, 555-594 (1995).

5. Paterson, G., Costen, M. L. \& McKendrick, K. G. Collisional depolarisation of rotational angular momentum: influence of the potential energy surface on the collision dynamics? Int. Rev. Phys. Chem. 31, 69-109 (2012).

6. Brouard, M. \& Vallance, C. (eds.) Tutorials in Molecular Reaction Dynamics (Royal Society of Chemistry, Cambridge, 2012).

7. Aoiz, F. J. et al. A new perspective: imaging the stereochemistry of molecular collisions. Phys. Chem. Chem. Phys. 17, 30210-30228 (2015).

8. Sharples, T. R. et al. Non-intuitive rotational reorientation in collisions of $\mathrm{NO}\left(\mathrm{A}^{2} \Sigma^{+}\right)$with $\mathrm{Ne}$ from direct measurement of a four-vector correlation. Nat. Chem. 10, 1148-1153 (2018). 
9. Wang, F., Lin, J.-S. \& Liu, K. Steric control of the reaction of CH stretch-excited $\mathrm{CHD}_{3}$ with chlorine atom. Science 331, 900-903 (2011).

10. Wang, F., Liu, K. \& Rakitzis, T. P. Revealing the stereospecific chemistry of the reaction of Cl with aligned $\mathrm{CHD}_{3}\left(\nu_{1}=1\right)$. Nat. Chem. 4, 636-641 (2012).

11. Perreault, W. E., Mukherjee, N. \& Zare, R. N. Quantum control of molecular collisions at 1 kelvin. Science 358, 356-359 (2017).

12. Van Leuken, J. J., Bulthuis, J., Stolte, S. \& Snijders, J. G. Steric asymmetry in rotationally inelastic state-resolved NO + Ar collisions. Chem. Phys. Lett. 260, 595-603 (1996).

13. Alexander, M. H. \& Stolte, S. Investigation of steric effects in inelastic collisions of NO(X) with Ar. J. Chem. Phys. 112, 8017-8026 (2000).

14. Gijsbertsen, A., Linnartz, H., Taatjes, C. A. \& Stolte, S. Quantum interference as the source of steric asymmetry and parity propensity rules in NO-rare gas inelastic scattering. J. Am. Chem. Soc. 128, 8777-8789 (2006).

15. de Miranda, M. H. G. et al. Controlling the quantum stereodynamics of ultracold bimolecular reactions. Nat. Phys. 7, 502-507 (2011).

16. Onvlee, J. et al. Imaging quantum stereodynamics through Fraunhofer scattering of NO radicals with rare-gas atoms. Nat. Chem. 9, 226-233 (2016).

17. Suits, A. G., Bontuyan, L. S., Houston, P. L. \& Whitaker, B. J. Differential cross sections for state selected products by direct imaging: Ar + NO. J Chem. Phys. 96, 8618-8620 (1992).

18. Kohguchi, H., Suzuki, T. \& Alexander, M. H. Fully state-resolved differential cross section for the inelastic scattering of the open-shell NO molecule by Ar. Science 294, 832-834 (2001). 
19. Lorenz, K. T. et al. Direct measurement of the preferred sense of NO rotation after collision with argon. Science 293, 2063-2066 (2001).

20. Gijsbertsen, A. et al. Differential cross sections for collisions of hexapole state-selected NO with He. J. Chem. Phys. 123, 224305 (2005).

21. Eyles, C. J. et al. Interference structures in the differential cross-sections for inelastic scattering of NO by Ar. Nat. Chem. 3, 597-602 (2011).

22. von Zastrow, A. et al. State-resolved diffraction oscillations imaged for inelastic collisions of NO radicals with He, Ne and Ar. Nat. Chem. 6, 216-221 (2014).

23. Vogels, S. N. et al. Imaging resonances in low-energy NO-He inelastic collisions. Science 350, 787-790 (2015).

24. Nichols, B. et al. Steric effects and quantum interference in the inelastic scattering of $\mathrm{NO}(\mathrm{X})$ + Ar. Chem. Sci. 6, 2202-2210 (2015).

25. Brouard, M. et al. Stereodynamics in $\mathrm{NO}(\mathrm{X})+$ Ar inelastic collisions. J. Chem. Phys. 144, 224301 (2016).

26. Eppink, A. T. J. B. \& Parker, D. H. Velocity map imaging of ions and electrons using electrostatic lenses: Application in photoelectron and photofragment ion imaging of molecular oxygen. Rev. Sci. Instrum. 68, 3477-3484 (1997).

27. Chandler, D. W. \& Houston, P. L. Two-dimensional imaging of state-selected photodissociation products detected by multiphoton ionization. J. Chem. Phys. 87, 1445-1447 (1987).

28. Eyles, C. J. et al. The effect of parity conservation on the spin-orbit conserving and spin-orbit changing differential cross sections for the inelastic scattering of $\mathrm{NO}(\mathrm{X})$ by Ar. Phys. Chem. Chem. Phys. 14, 5420-5439 (2012). 
29. Alexander, M. H. A new, fully ab initio investigation of the $\mathrm{NO}\left(\mathrm{X}^{2} \Pi\right)-$ Ar system. I. Potential energy surfaces and inelastic scattering. J. Chem. Phys. 111, 7426-7434 (1999).

30. Eyles, C. J. et al. Fully $\Lambda$-doublet resolved state-to-state differential cross-sections for the inelastic scattering of $\mathrm{NO}(\mathrm{X})$ with Ar. Phys. Chem. Chem. Phys. 14, 5403-5419 (2012).

31. McCurdy, C. W. \& Miller, W. H. Interference effects in rotational state distributions: Propensity and inverse propensity. J. Chem. Phys. 67, 463-468 (1977).

32. Brouard, M. et al. Integral steric asymmetry in the inelastic scattering of $\mathrm{NO}\left(\mathrm{X}^{2} \Pi\right)$. J. Chem. Phys. 146, 014302 (2017).

33. Khare, V., Kouri, D. J. \& Hoffman, D. K. On $j_{z}$-preserving propensities in molecular collisions. I. Quantal coupled states and classical impulsive approximations. J. Chem. Phys. 74, 2275-2286 (1981).

Acknowledgements The support of the UK EPSRC (to M.B. via Programme Grant EP/L005913/1), and the Spanish Ministry of Science and Innovation (Grant No. CTQ2015-65033-P to F.J.A.) is gratefully acknowledged. P.G.J. acknowledges funding by Fundación Salamanca city of culture and knowledge (programme for attracting scientific talent to Salamanca). We would like to thank Professor Steven Stolte for many stimulating discussions throughout the course of some of the research described here.

Author contributions The research project was conceived by M.B., C.G.H. and F.J.A. The experiments were performed by V.W. and C.G.H., and the data analysis was carried out by V.W. The theory was developed by M.B., V.W., P.G.J., and F.J.A., with calculations performed by V.W. and P.G.J. The paper was written by M.B. and F.J.A., with all authors contributing to discussions about the content of the paper.

Competing Interests The authors declare that they have no competing financial interests. 
Additional Information Supplementary Information is linked to the online version of the paper at www.nature.com/nchem.

Reprints and permissions information is available at npg.nature.com/reprints.

Correspondence and requests for materials should be addressed to M.B. (mark.brouard@chem.ox.ac.uk). 


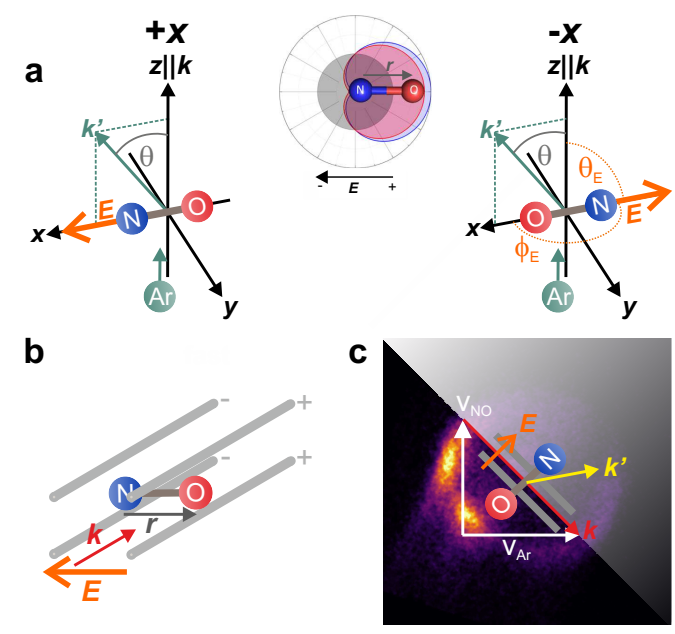

Figure 1: Schematic of the experimental configuration for bond-axis orientation measurements. a, The static field directions $\left(\theta_{E}, \phi_{E}\right)$ are defined in the scattering frame, in which the initial relative velocity vector, $\boldsymbol{k}=\boldsymbol{v}_{\mathrm{Ar}}-\boldsymbol{v}_{\mathrm{NO}}$, lies parallel to the $z$-axis, the $(+x)(+z)$-quadrant contains $\boldsymbol{k}$ and $\boldsymbol{k}^{\prime}\left(=\boldsymbol{v}_{\text {Ar }}^{\prime}-\boldsymbol{v}_{\mathrm{NO}}^{\prime}\right)$, the final relative velocity vector, and $\theta$ denotes the scattering angle. The $+x$ (left) and $-x$ (right) field orientations correspond to $\theta_{E}=\pi / 2, \phi_{E}=0$ and $\theta_{E}=\pi / 2, \phi_{E}=\pi$, respectively; the angles are indicated for the $-x$ configuration. The initial isotropic bond-axis probability distribution without a static field is shown as a grey shaded circle in the top inset. The bond-axis probability distribution at the experimental field, $\boldsymbol{E}$, is shown as the red shaded area and at infinite field as the blue shaded area. The orientation of the NO molecule represents the most probable configuration. b, A static electric field, created by a set of four rods, preferentially orients the NO bond-axis, $\boldsymbol{r}$, antiparallel to the field, $\hat{\boldsymbol{E}}$. c, The Newton diagram for the $+x$-orientation, assuming scattering occurs in the plane of the molecular beams, is overlaid with the experimental ion image for the $\Delta j=9$ transition. The orientation label " $+x$ " refers to the lower left, unshaded portion of the image, where the signal intensity corresponding to the scattered NO molecules opposes $\boldsymbol{k}^{\prime}$ (the direction of the scattered Ar atoms). 


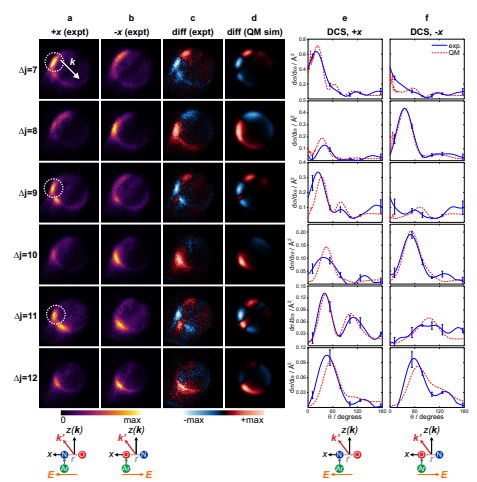

Figure 2: Bond-axis orientation resolved experimental ion images and differential cross sections (DCSs) compared to QM theoretical results. a-d, Experimental images for the $+x(\mathbf{a})$ and $-x$-orientation (b), corresponding to repulsive scattering from the $\mathrm{N}$-side and $\mathrm{O}$-side, respectively, alongside experimental (c) and QM simulated (d) difference images, for $\Delta j=7$ to 12 transitions into the final $e \Lambda$-doublet. The $+x$ and $-x$-orientation labels on the ion images in $\mathbf{a}$ and $\mathbf{b}$ refer to NO scattering in the plane of the molecular beams (coincident with the image plane) into the intense lower left region of the image (see Fig. 1c). For odd $\Delta j$, the prominent peak corresponding to forward scattering (of NO with respect to its initial motion) is highlighted with a dashed circle. e,f, The differential cross sections extracted from the experimental images (blue continuous lines) are compared to QM calculations (red dashed lines). The error bars in the experimental data correspond to one standard deviation, calculated from individual fits to the bottom left half, the top right half, and the whole image. The panels in the bottom row illustrate the simplified configuration relevant to in-plane scattering into the bottom left intense portion of the images, with the most probable orientation of the bond-axis also included. The preference for $+x /-x$ orientation alternates between odd and even $\Delta j$ transitions; this is particularly evident in the difference images (c,d), in which blue/purple in the lower left half of the images represents a $-x$-axis and yellow/red a $+x$-axis preference (and vice versa in the top right half of the images). 

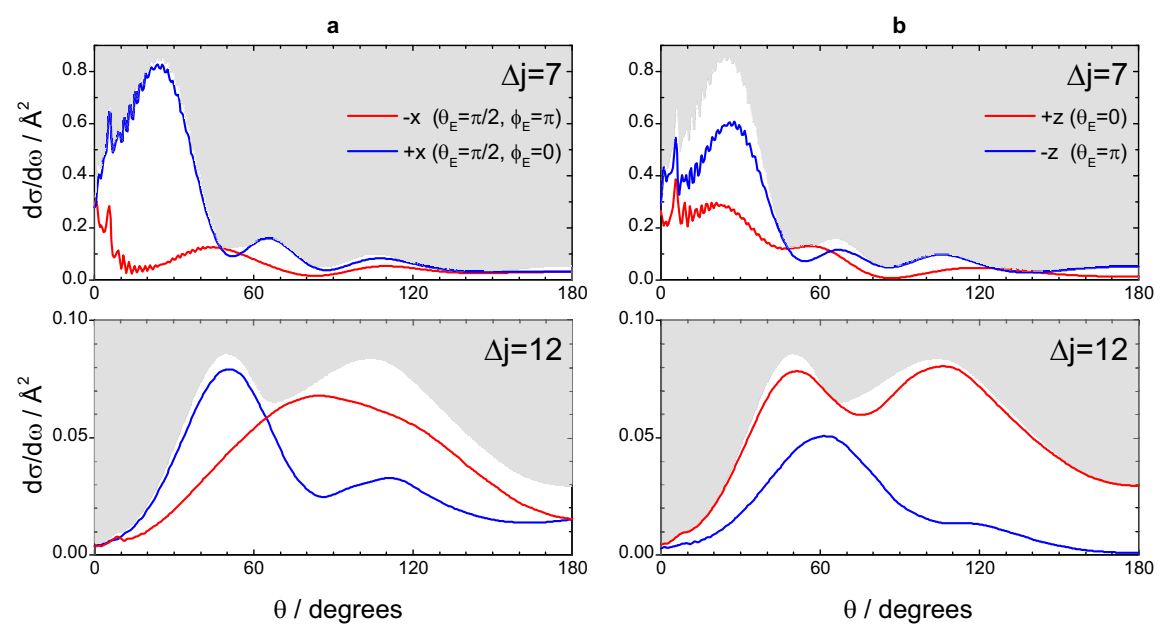

Figure 3: Comparison of the differential cross sections (DCSs) for side-on and end-on orientation. a The maximised DCSs (white area) are shown with the $+x(\mathrm{~N}$-side, blue lines) and $-x$ (O-side, red lines) oriented DCSs for $\Delta j=7$ and 12. b Same as in a, but showing the DCSs for $+z$ (O-end collisions, red lines) and $-z$ (N-end collisions, blue lines). (A more complete set of data is shown in Supplementary Fig. 5.) The data are calculated in the limit of infinite static field strength in which the initial $e$ and $f \Lambda$-doublet states are fully mixed such that the bond axis is maximally oriented antiparallel to the field direction. The DCSs were maximised with respect to $\theta_{E}$ and $\phi_{E}$, the direction of the static field (see Fig. 1a). As can be seen, for $\Delta j=7$, side-on orientation $(+x$ in this case) almost maximises the DCS around the prominent forward peak, whilst for $\Delta j=12$, the end-on orientation (O-end $(+z)$ in this case) is close to the maximum DCS in the backward scattered region. 

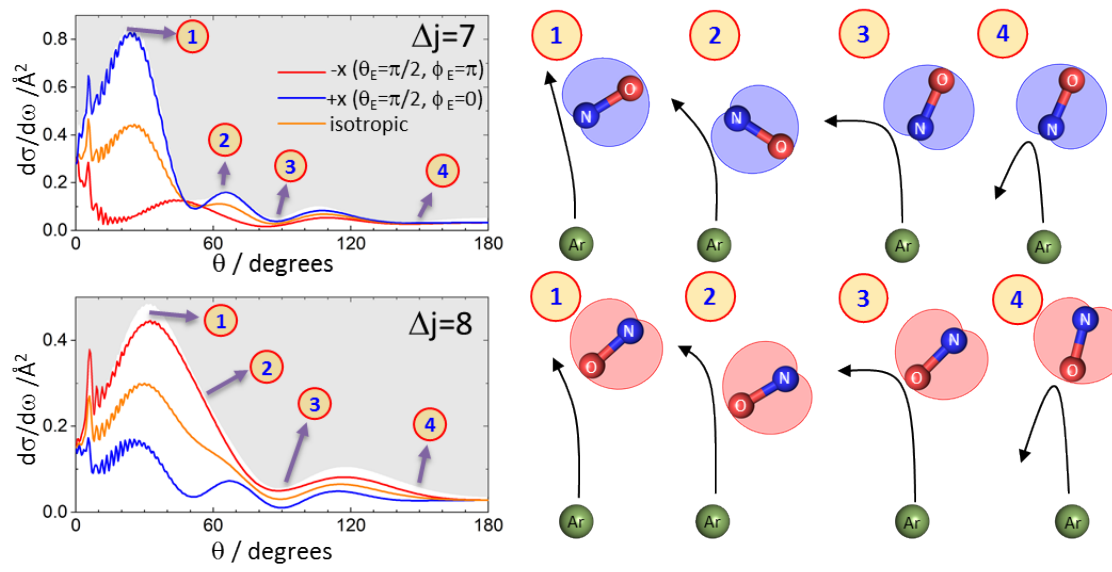

Figure 4: Illustrations of the scattering mechanism derived from the initial bond-axis oriented

calculations. a As for Fig. 3a, but showing the QM isotropic DCSs (equivalent to the average of the $+x$ and $-x$, or $+z$ and $-z$, oriented DCSs (shown as the orange line)) for $\Delta j=7$ and 8 together with the $+x$ (blue lines) and $-x$ (red lines) bond-axis oriented DCSs. b Sketches of the mechanisms that maximise the DCSs at selected scattering angles, with the corresponding QM calculated initial bond-axis probability distributions shown by the shaded areas (red for Oend/side preferred collisions, and blue for $\mathrm{N}$-end/side preferred collisions). Note the opposite preference for odd and even $\Delta j$ transitions, which is due to quantum interference between the scattering amplitudes of the two initial $\Lambda$-doublet states. The cartoons illustrate that nearly side-on geometries dominate in the forward scattered region (cartoons 1,2), whereas geometries that are more end-on dominate in the backward scattered region (cartoons 3,4). 